\title{
Perceptually-based Color Assignment
}

\author{
Hye-Rin Kim ${ }^{1}$, Min-Joon Yoo ${ }^{1}$, Henry Kang ${ }^{2}$, and In-Kwon Lee ${ }^{1}$ \\ ${ }^{1}$ Dept. of Computer Science, Yonsei University, South Korea \\ ${ }^{2}$ Dept. of Math. and Computer Science, University of Missouri - St. Louis, USA
}
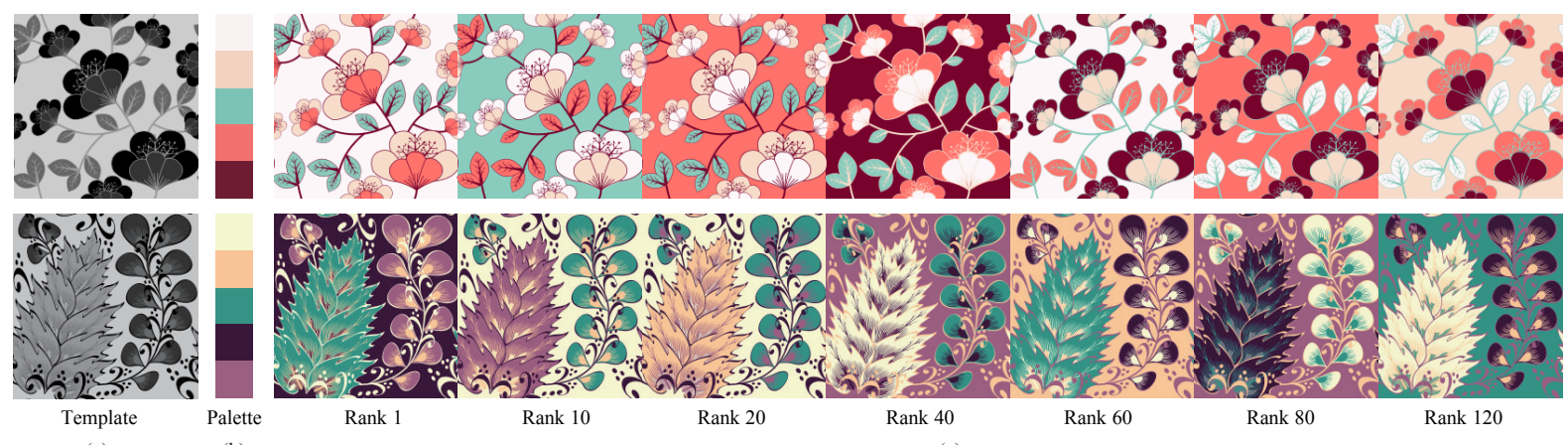

(b)

(c)

Figure 1: Given a template made up of disjoint image segments (a) and a color set (b), our method evaluates and ranks the assignment of colors to the segments. (c) shows sample results from 120 possible assignments and their ranks.

\begin{abstract}
Color assignment is a complex task of incorporating and balancing area configuration, color harmony, and user's intent. In this paper, we present a novel method for automatic color assignment based on theories of color perception. We define color assignment as an optimization problem with respect to the color relationships as well as the spatial configuration of input segments. We also suggest possible constraints that are suitable for taskspecific purposes and for enhancing visual appeal. Our colorization scheme is useful in many applications such as infographics, computer-aided design, and visual presentation. The user study shows that our method generates perceptually pleasing results over a variety of data sets.
\end{abstract}

Categories and Subject Descriptors (according to ACM CCS): I.3.0 [Computer Graphics]: Graphics UtilitiesGraphics editors, H.5.2 [Information Interfaces and Presentation]: User Interfaces-Screen design

\section{Introduction}

Color is an essential element of many facets of life: from selecting colors for one's clothes, cars, furniture, webpages, to designing, decorating, and conveying ideas, materials, and even emotions. Computer-aided colorization may assist humans in making these decisions and improving the visual quality of the outcome. The primary challenges in many colorization applications include, first, selecting a color palette that best represents the idea or purpose of the content be- ing colorized [MSK04, OAH11, Ado13, COL13]. The usual next step is to assign the selected colors to the appropriate image partitions or segments. This is an equally (if not more) important and difficult task, for which one must consider how the input segments are shaped and composed, what colors are to be assigned and where in order to maximize the desired goals, and how visually appealing the outcome would be. We are particularly interested in solving the problem of coloring a $2 \mathrm{D}$ pattern template using a given color palette [SMV*10, OAH11, LRFH13, MLM13], which 
we face in many practical application scenarios. We present a simple and intuitive approach for assigning colors to a set of uncolored image segments, based on existing rules discovered in the area of color perception. We build an objective function to evaluate and rank the assignment of colors by taking into account the color relationship in both spatial and range domains. The proposed method is designed to provide an efficient and intuitive process of colorization as well as visually pleasing results, as will be verified by our perceptual study.

The main contributions of this paper are thus summarized as follows:

- A novel, rule-driven method for assigning colors to uncolored image segments

- Additional constraints to help enhance the quality of output in accordance with the purpose and intent of colorization

- A crowd-sourcing-based user study to evaluate the proposed scheme against other methods

\section{Related work}

\subsection{Color harmony}

Color theory has a long history, dating back to Goethe [Goe10] followed by a plethora of subsequent discoveries on color harmony, balance, and perception. One of the well-known rules states that "colors having similar hues or complementary hues are harmonious". A variety of algorithms have been proposed to create harmonious color compositions based on this principle. Furthermore, several groups of researchers have developed computational models of color harmony [NS09, Nem07, SP11, CoSG*06, SBS10]. These models are often derived from perceptual/psychological experiments and represented in the form of mathematical formula to quantify color harmony. Ou and Luo [OL06] established a color harmony model based on a perceptual experiment with human subjects. We employ their model to perform color assignment in such a way that the color harmony is maximized.

\subsection{Luminance contrast}

Luminance contrast is a basic image property which measures the difference of lightness between foreground and background, or between two neighboring image components. Increasing the contrast often enhances the legibility of the image content, for instance the use of black text on white background in books, documents, and visual acuity assessments. It is also widely used for image enhancement [MI06, XZTZ10], saliency detection [MZ03, CZM*11] and object recognition [ADF10, FGYZ12]. In particular, Ma and Zhang [MZ03] explained why luminance contrast can be more important in terms of visual perception than other properties such as color, texture, and size, and introduced a new saliency map generation method based on it. We have employed [MZ03]'s insight and incorporate global luminance contrast as part of our objective function.

\subsection{Color Assignment}

There have been several works on assigning colors to uncolored image segments. Sauvaget et al. [SMV*10] assigned colors to a segmented image based on "proportional harmony" that seeks a quantitative balance between colors with respect to area proportions. Since the color assignment in this approach is a function of hue and area proportion, somewhat consistent and predictable results are expected (e.g., violet and blue tend to go to large segments, and yellow and orange to small segments). We look for a more general coloring scheme that provides a host of control parameters to incorporate user's intent. Moretti et al. [MLM13, MML13] introduced a software tool called "Colour Harmonizer" that takes user's color selection from which to generate color schemes for target templates. Its main focus however is on a rather specific template type such as webpages. While these systems are similar to our approach in using predefined colors, our method is simpler and is capable of handling a broader range of template configurations.

Recently, a data-driven approach was introduced that creates colorization of segmented images from a user-provided set of examples. O'Donovan [OAH11] suggested a datadriven quantitative model that evaluates the compatibility of colors by predicting ratings for a color palette of five colors. Lin et al. [LRFH13] presented a probabilistic model to solve color selection problem as well as color assignment problem. Their model recommends various colorization possibilities of a given 2D pattern template, using sample data from COLOURlovers [COL13] and learning the model by analyzing geometric information of template and color properties. In contrast, our method does not require a complex process of data-driven machine learning, but leverages widely accepted rules in color theories that are intuitive to understand and easy to implement.

\section{Proposed method}

\subsection{Problem formulation}

We define template as a segmented image, where each segment has been assigned a certain gray-level intensity. An area is a collection of one or more segments that share the same input gray-level intensity (see Figure 2). Given an area set $A=\left\{A_{1}, \cdots, A_{N}\right\}$ in the input template and a color palette $C=\left\{C_{1}, \cdots, C_{M}\right\}$, our goal is to find a mapping from $A$ to $C$ that maximizes color harmony and luminance contrast.

Formally, we define the color assignment problem as finding a mapping function $\sigma$ s.t. $\sigma\left(A_{i}\right)=C_{j}, i \in[1 . . N], j \in$ 
(a)

(b)
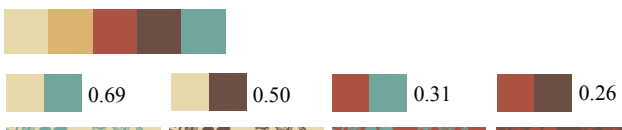

26
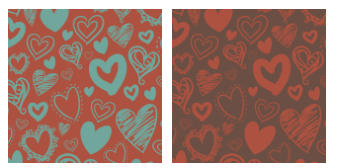

(c)
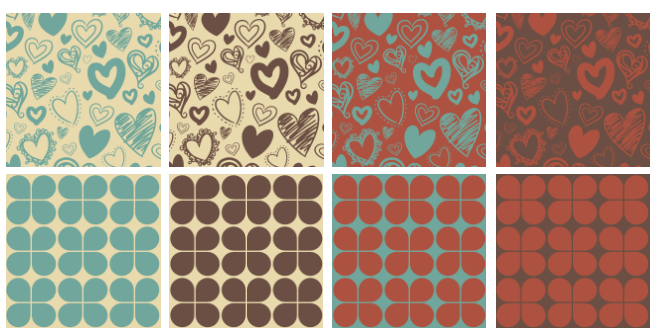

(d)
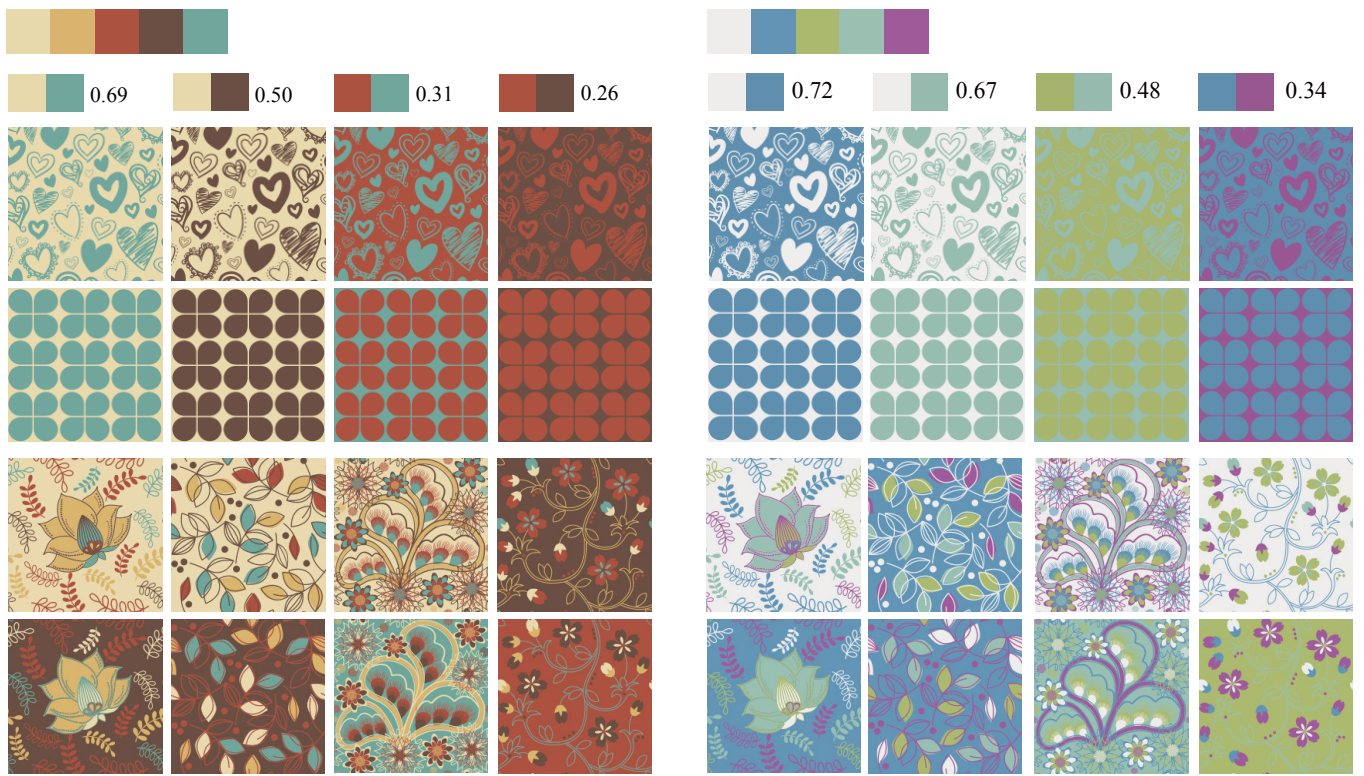

Figure 3: The effect of the color harmony term. Given palette (a), the harmony of any color pair can be evaluated and converted into a numeric value. (b) shows some example color pairs along with their binary colorizations in $(c)$. (d) and (e) show example full color assignments using the given palette, where $(d)$ is ranked higher (better) than $(e)$ in terms of color harmony.

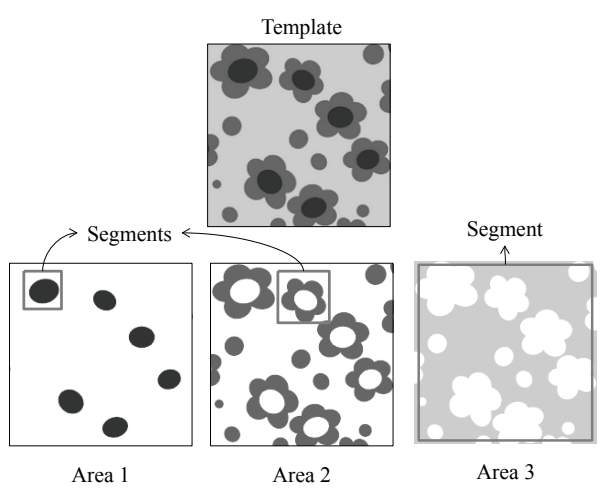

Figure 2: A template is composed of disjoint areas, and an area is a collection of one or more segments having the same gray-level intensities. In our experiments, we use a template with five areas and a color palette with five colors.

$[1 . . M]$, which maximizes the following objective function:

$$
E=\sum_{A_{i}, A_{j} \in A} E_{h}\left(A_{i}, A_{j}, \sigma\right)+E_{c}\left(A_{i}, A_{j}, \sigma\right)+\lambda \cdot E_{a}\left(A_{i}, A_{j}, \sigma\right)
$$

The first term $E_{h}$ measures the color harmony (Section 3.2), the second term $E_{c}$ measures the luminance contrast of each area pair (Section 3.3), and the last term $E_{a}$ controls the additional constraints, if any (Section 3.4). In our experiments we set $\lambda=10$ for each additional constraint (Figure $10)$, and $\lambda=0$ when there is none.

\subsection{Color harmony}

There are several ways to estimate the harmony or compatibility of color combination [SBS10,OAH11]. In our method, we employ a color harmony model based on Ou and Luo's psychological experiments [OL06]. They introduced a quantitative model $\mathcal{H}$ which measures the color harmony based on three factors: chromatic, lightness, and hue effect, in CIELab color space. They conducted the experiments in the limited environment where the subjects were allowed to rate the harmony of two color combinations between -5 and 5 . We adapt this model so that the colors are assigned while maximizing the color harmony of every combination of two constituent colors:

$$
E_{h}\left(A_{i}, A_{j}, \sigma\right)=w_{i j} \cdot \mathcal{H}\left(C_{p}, C_{q}\right)
$$

where $\sigma\left(A_{i}\right)=C_{p}$ and $\sigma\left(A_{j}\right)=C_{q}$. We normalize the harmony values to range between 0 and 1 . See Appendix for more details on $\mathcal{H}$.

For each pair of areas $A_{i}$ and $A_{j}$, we compute the harmony of their respective colors $C_{p}$ and $C_{q}$. The weight $w$ is defined as the total length of the border between the areas. That is, we set $w_{i j}$ as the number of pixels on the border between $A_{i}$ 


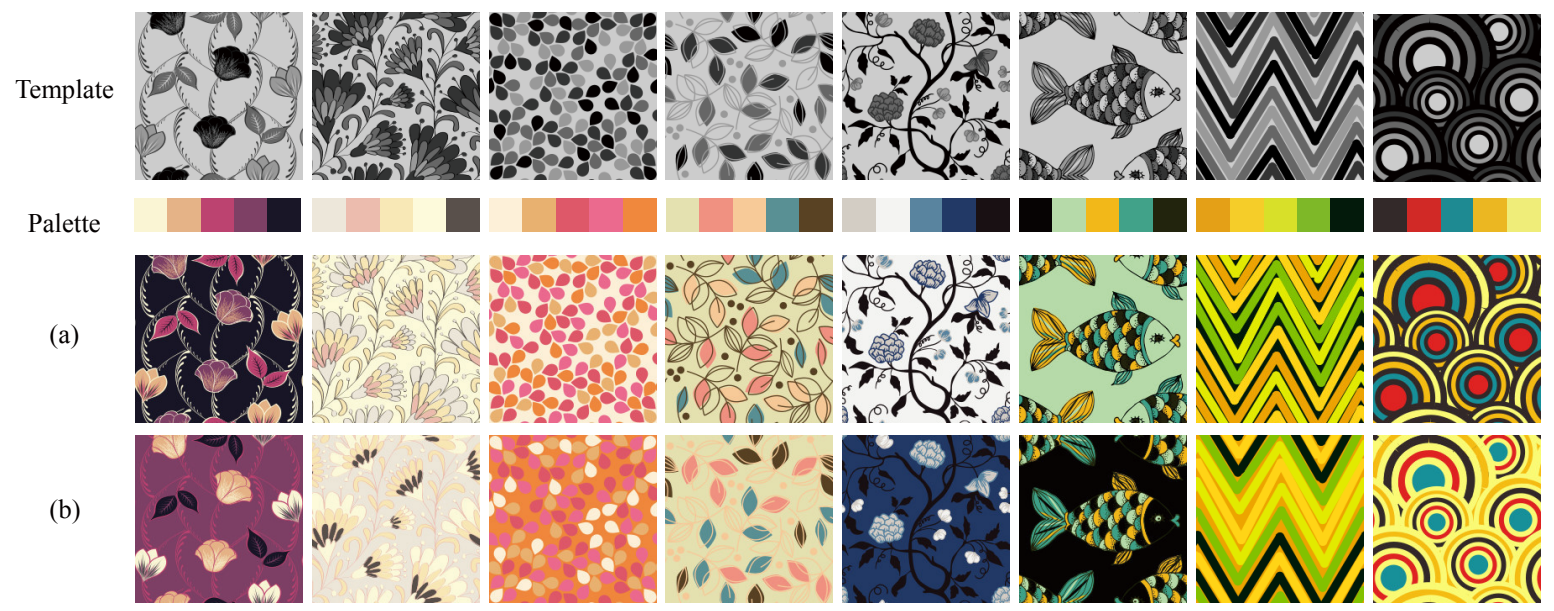

Figure 5: Color assignment (a) with and (b) without the contrast term. (a) shows clearer distinction between adjacent areas.

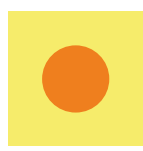

(a)

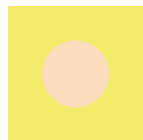

(b)

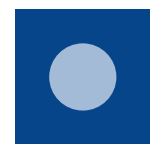

(c)

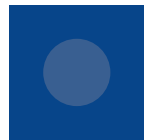

(d)

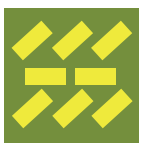

(e)

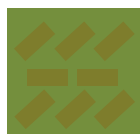

(f)

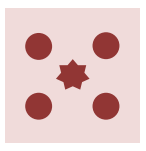

(g)

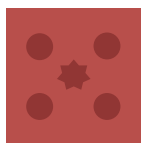

(h)
Figure 4: Contrast draws attention even more so than absolute color, texture, and shape.

and $A_{j}$. This encourages stronger harmony between adjacent areas. Figure 3 demonstrates the effect of our color harmony term.

\subsection{Luminance contrast}

Contrast is an important parameter in visual perception [Lam91]. It is known that when people see an object, the human visual system is highly sensitive to its contrast against the background, but not so much to its absolute color, texture, and shape. Figure 4a-d shows that regardless of the colors used for the foreground and the background, strong enough contrast is needed to draw the viewer's attention. Similarly, without enough contrast, texture or shape information can quickly lose its appeal and become somewhat ambiguous (Figure 4e-h). Based on these observations, [MZ03] suggested that areas with high contrast are more likely to attract human attention and help distinguish scene components. We thus define the contrast term as

$$
E_{c}\left(A_{i}, A_{j}, \sigma\right)=w_{i j} \cdot \mathcal{D}\left(C_{p}, C_{q}\right)
$$

where $\sigma\left(A_{i}\right)=C_{p}, \sigma\left(A_{j}\right)=C_{q}$ and $\mathcal{D}\left(C_{p}, C_{q}\right)$ is the luminance distance of two colors $C_{p}$ and $C_{q}$ for which we use $L_{2}$ distance on the $\mathrm{L}$ channel of CIELab color mode. The weight $w_{i j}$ is calculated in the same way as in Equation (2).

Given the image template and the color palette, the contrast term looks to maximize the sum of lightness distance between all assigned color pairs. The weight $w$ promotes clearer distinction between adjacent areas. Figure 5 shows examples with and without the contrast term.

\subsection{Additional constraints}

As will be shown in Section 4, the aforementioned formulation with the default parameter setting generates satisfactory results on a variety of data sets. Here we introduce some additional constraints to meet application-specific needs. We adapt $E_{a}=\left\{E_{\text {stab }}, E_{\text {cont }}\right\}$ term to our model according to the type of constraints : $E_{a}=E_{\text {stab }}$ for stability and $E_{a}=E_{\text {cont }}$ for continuity constraint. Similarity constraint is implemented as a hard constraint.

Stability [Bul07] suggested that when colors are arranged vertically, it is desirable to have darker colors on the lower side, providing viewer with the sense of gravitational stability. Such a constraint may be given by:

$$
E_{s t a b}\left(A_{i}, A_{j}, \sigma\right)=w_{i j} \cdot\left(\frac{1}{L_{i} \cdot y\left(A_{i}\right)}+\frac{1}{L_{j} \cdot y\left(A_{j}\right)}\right)
$$

where $y(A)$ is the vertical center of area $A$ and $L_{k}$ is the lightness of the color $\sigma\left(A_{k}\right)$. We normalized the image height to range from 0 (lowest position) to 1 (highest position).

Continuity Continuity rule in Gestalt laws states that people tend to perceive shapes as a whole when they appear to 
form a continuous path [Wer38]. As a variant of this rule, we suggest a constraint on space-color continuity. Note that continuity is the opposite of contrast, in that we encourage adjacent areas to have similar luminance values.

$$
E_{\text {cont }}\left(A_{i}, A_{j}, \sigma\right)=w_{i j} \cdot\left(1-\mathcal{D}\left(C_{p}, C_{q}\right)\right)
$$

Similarity According to the law of grouping in Gestalt theory, people tend to perceive elements that have similar shapes, sizes, or colors as a group [Wer38]. Therefore, we may optionally enforce that areas of similar shapes and/or sizes be assigned similar colors in the palette.

\subsection{Optimization}

With Equation (1) all set as described above, our goal is to find a mapping $\sigma_{\max }$ that maximizes $E$.

$$
\sigma_{\max }=\arg \max _{\sigma} E
$$

We can simply find the globally optimal solution that maximizes the energy among all possible colorings. In this process, non-optimal solutions are also ranked in terms of their objective function values. In case there is a large number of colors and/or areas which makes it impractical to compute the globally optimal solution, a stochastic optimization process or a graph-based labeling technique may be used instead [GG84, BVZ01].

\section{Results}

This section presents test results of our method on certain application scenarios. All templates used consist of five areas, each of which may further decompose into multiple segments. As for color palettes, we used several fivecolor themes collected from Adobe Kuler [Ado13] and COLOURlovers [COL13].

Figure 1 and 6 show our test results on a series of uncolored templates available at COLOURlovers. For each coloring result, its ranking number reflects where it stands among all possible colorings in terms of maximizing the energy function. The number is assigned as inversely proportional to the energy function value, which means Rank 1 represents the best solution, and Rank 120 the worst. Note that the Rank 1 results generally meet the aforementioned criteria on color harmony and contrast.

Figure 7 shows colorization of a 3D scene. We apply our method on the objects in the scene abstracted and classified into five $2 \mathrm{D}$ areas. The final rendering is then obtained by adding details back on the scene.

Figure 8 shows our test results on web page colorization, which is a common application in the realm of color assignment [MLM13]. Web designers typically use three to five major colors in their design. For our test, we use a standard web page template with five areas (background, main body, header text, menu buttons, and button text).
Figure 9 shows coloring of graph layouts such as pentagonal, line, and bar graphs. Our method allows users to pick and choose from multiple coloring choices.

Figure 11 shows our test results on a template with text. In general, increasing luminance contrast between text and background improves readability [Sto12], which is consistent with how our model is designed. When it is necessary to fix a color on a particular area, it can be enforced as a hard constraint. Figure 12 shows an example.

In general, there is no "ground truth" in the task of color assignment. We thus performed three perceptual studies to evaluate our method.

Experiment 1 The first perceptual study was conducted in order to evaluate our method against [LRFH13], one of the state-of-the-art techniques. We first selected fourteen templates used in Lin et al.'s method, then for each of them we collected four color palettes and color assignments recommended by their method. On each of these 56 combinations of templates and color palettes, we ran our method and generated 56 colorings that marked Rank 1 in each case.

For each of these 56 template-palette pairings, we provided human subjects with two images: our result and Lin et al.'s result. The two images were placed in random order (left and right). In each case, the subjects were asked to select one of them based on their preference (using two-alternative forced choice design). The subjects were recruited on Amazon's Mechanical Turk, and a total of 100 people participated in the experiment. For most subjects, the experiment took just a few minutes. In total, the subjects gave 5589 evaluations (11 evaluations were missing from 10 participants). Figure 13 contains some results as well as how they were ranked statistically by the subjects. Additional results can be found in the supplementary material.

Table 1 summarizes the preference analysis derived from this perceptual study. The 'Model' preference describes which of the two models was statistically preferred over the 56 cases. The result shows that our model was statistically preferred by the subjects 35 out of 56 times. As for the 'Subject' preference, we summed the number of subjects that chose our model over Lin et al.'s in each of the 56 cases, then computed the average. We did the same for Lin et al.'s model too. As shown in the table (second row), over half of the people on average preferred our results. The results created by our model are significantly preferred against the results from Lin. et al's model ( $p<0.05$ using one tailed t-test).

Experiment 2 We conducted another user study to evaluate our additional constraints described in Section 4 . Table 2 shows the preference rankings of colorings generated using the constraints. A total of 35 subjects participated in the experiments through Amazon's Mechanical Turk. For testing the continuity and stability constraints, we showed the subjects five results obtained with the respective constraint, 


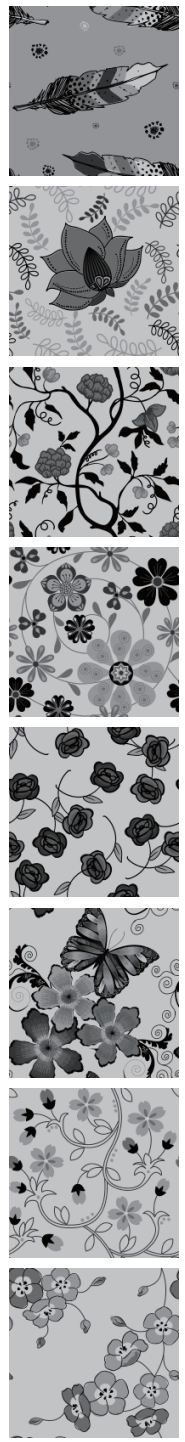

Template
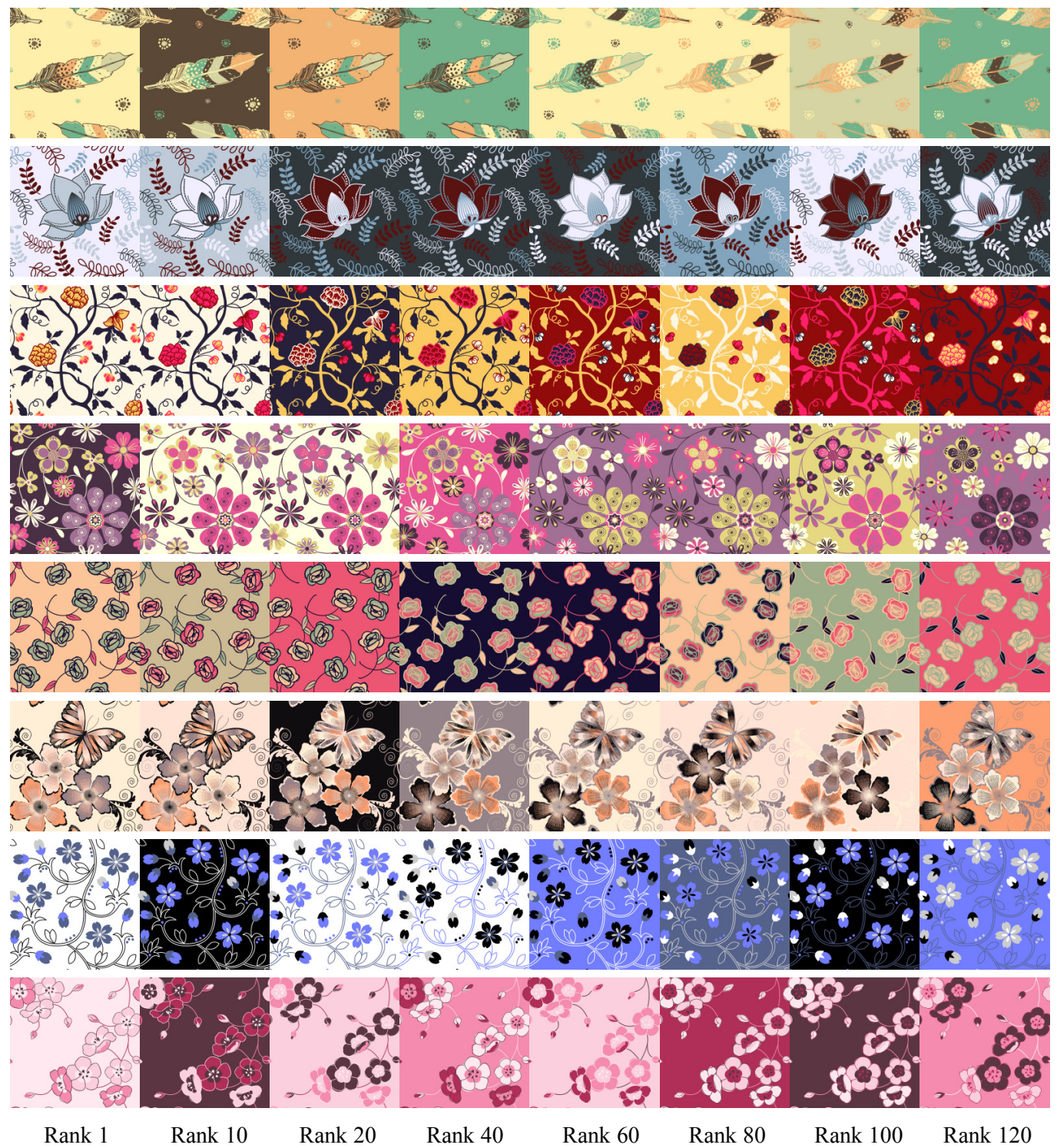

Figure 6: Input templates and various colorization results with their ranks (lower number means more recommended).

\begin{tabular}{lccc}
\hline Preference & Ours & Lin et al. & Total \\
\hline Model & $62.5 \%(35)$ & $37.5 \%(21)$ & $100 \%(56)$ \\
Subject & $56.48 \%$ & $43.52 \%$ & $100 \%$ \\
\hline
\end{tabular}

Table 1: Preference analysis from the perceptual study. The number in parentheses (first row) indicates how many times each model was statistically preferred.

ranked 1, 30, 50, 90 and 120 . The subjects were then asked to select one of them based on their preference. We used 10 different template-palette pairings including the ones in Figure 10 and therefore obtained 350 responses for each constraint. It turns out that people prefer Rank 1 results for both types of constraints, indicating that the use of constraints does contribute to enhancing the visual appeal. For similarity constraint, we showed two pictures, one with constraint and one without, then asked them to pick one they liked better. As shown in Table 2, the majority of the participants preferred the results with the constraint. 


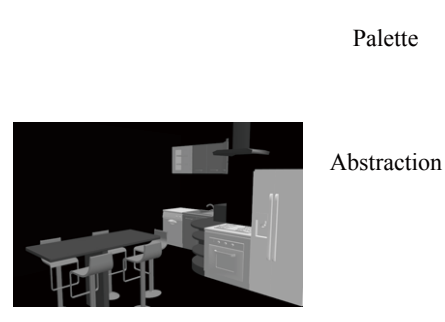

Suggestion

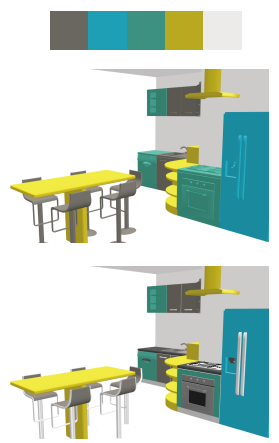

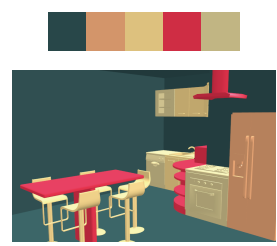
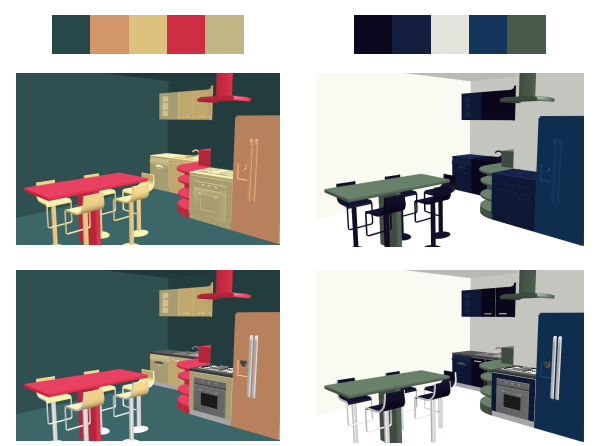

Figure 7: Three colored results of 3D virtual scene for interior design. Left: Uncolored template. Top: Color palette. Middle: Colorings on an abstracted scene. Bottom: Final renderings.
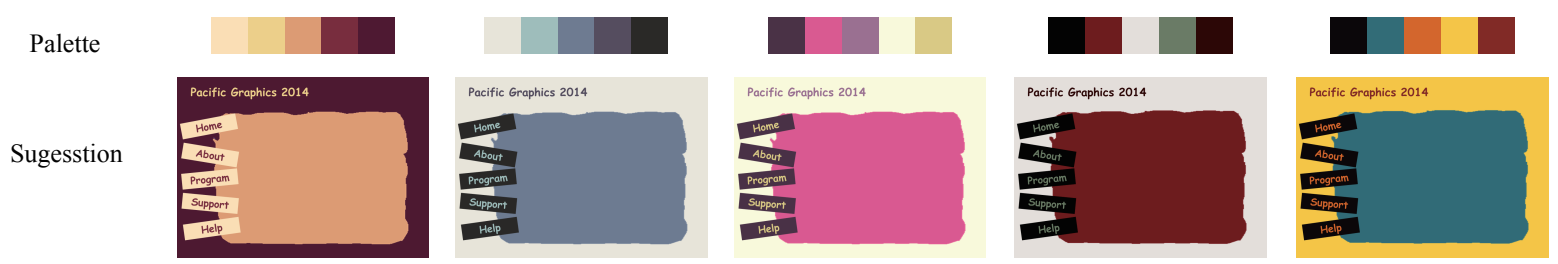

Figure 8: Web page coloring on a layout composed of five areas. A similarity constraint was used to ensure that the five menu buttons and the text on them have the same colors.
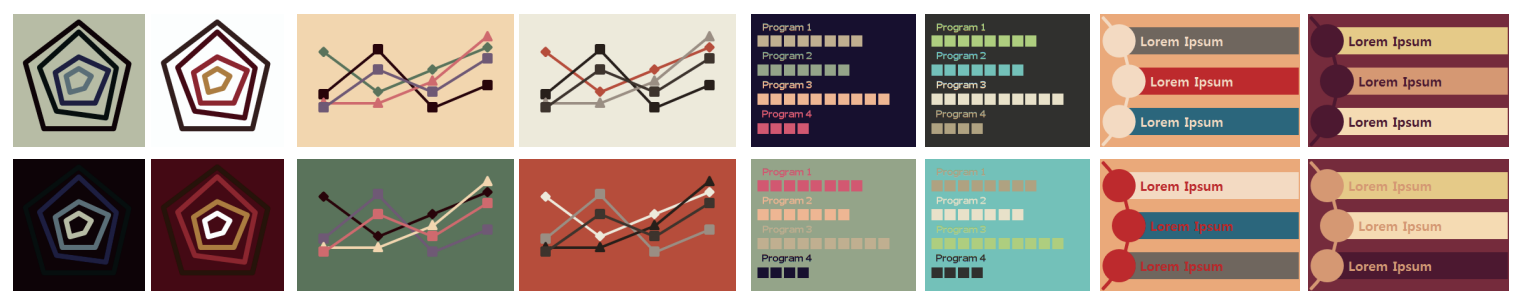

Figure 9: Coloring of graph layouts. Rank 1 (top) and Rank 120 (bottom) results for each graph layout.

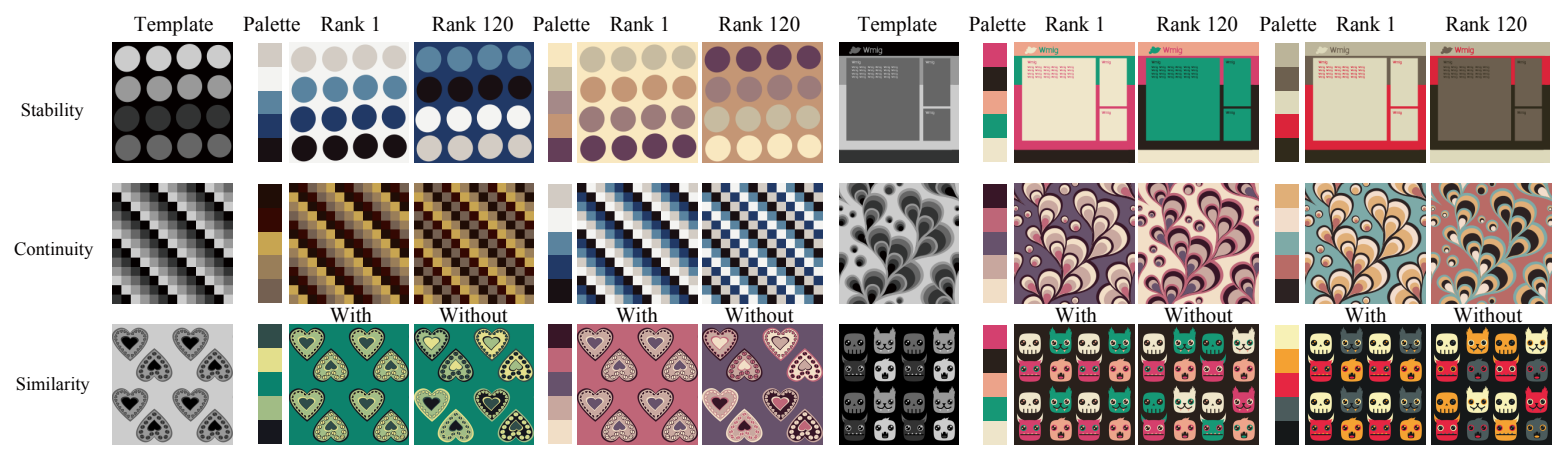

Figure 10: Example usage of the additional constraints. Left: Uncolored template for each constraint. Middle: Color palette. Right: Coloring results using constraints and their ranks. In case of similarity constraint, the examples show the side-by-side comparisons of the coloring results obtained with and without the constraint. 


\begin{tabular}{l|c|c|c|c|c}
\hline \multirow{2}{*}{} & \multicolumn{5}{|c}{ Ranks } \\
\cline { 2 - 6 } & 1 & 30 & 50 & 90 & 120 \\
\hline Continuity & $27 \%$ & $23 \%$ & $16 \%$ & $20 \%$ & $14 \%$ \\
\hline Stability & $25 \%$ & $16 \%$ & $17 \%$ & $20 \%$ & $22 \%$ \\
\hline \multirow{2}{*}{ Similarity } & With & \multicolumn{5}{|c}{$32 \%$} \\
\cline { 2 - 6 } & $68 \%$ & \multicolumn{5}{|c}{$32 \%$ t } \\
\hline
\end{tabular}

Table 2: Preference rankings of colorings generated using additional constraints. Rank 1 results were voted first for continuity and stability constraints. For similarity constraint, results obtained with the constraint were preferred over the ones without.

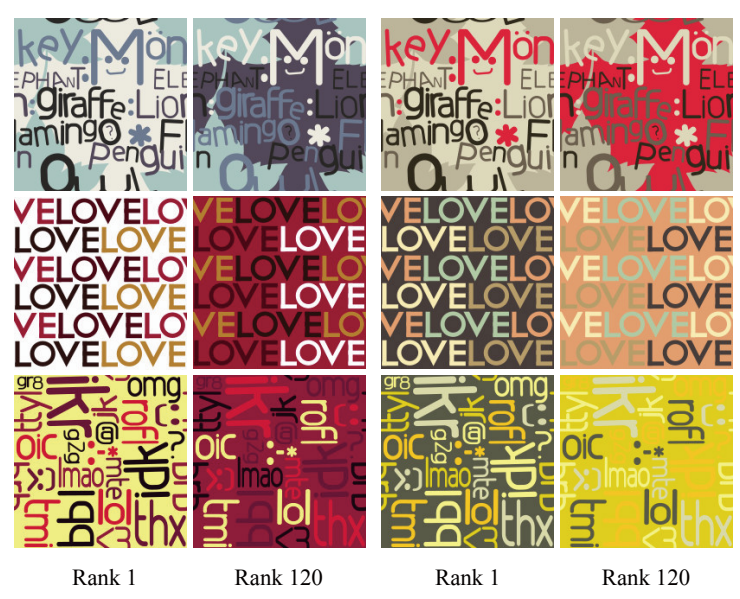

Figure 11: Coloring of templates containing text.

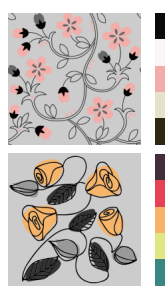

(a)

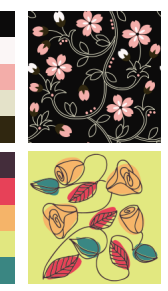

(b)

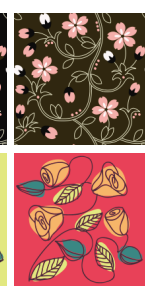

(c)

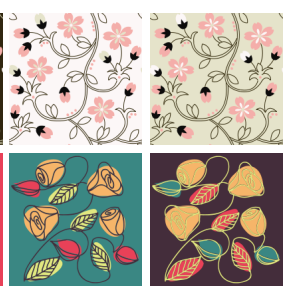

Figure 12: Fixing color on a specified area. Pink (top) and orange (bottom) are designated to the flowers.

Experiment 3 We have also compared our results with the manually generated colorizations. We collected 31 pattern templates from COLOURlovers and top ten most-voted colorings for each of those templates that were manually generated by the users. We then extracted colors from each of the top ten human colorings, and ran our method to colorize the same template using those colors. Figure 15 shows some results of this experiment. Additional results can be found

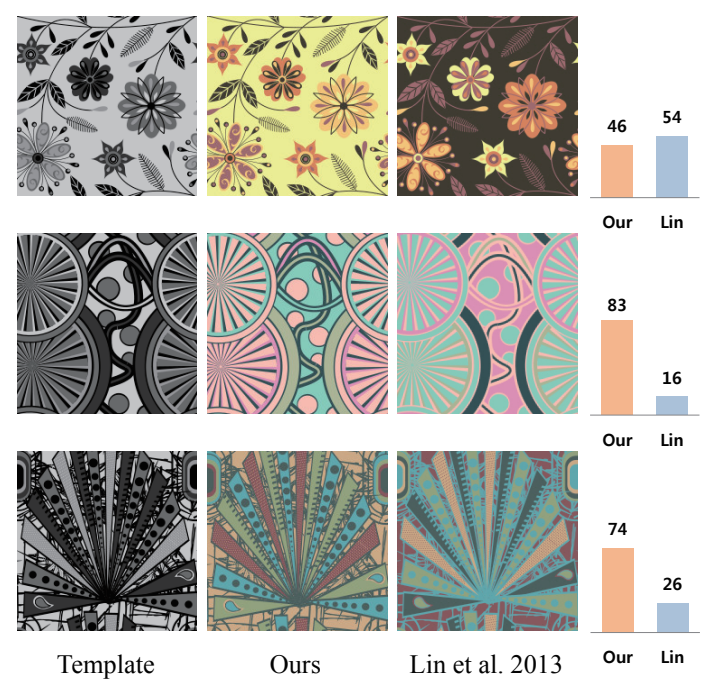

Figure 13: Comparison with Lin et al. For each template, the subjects selected either Lin et al.'s coloring or ours. The graph on the right represents the number of people who chose ours vs. Lin et al.'s.

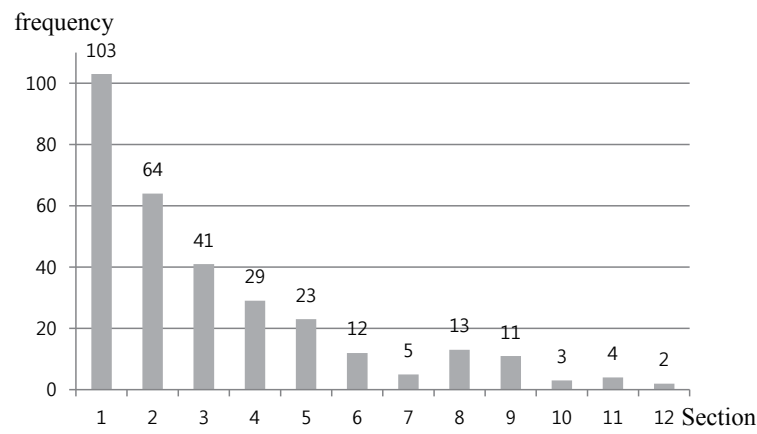

Figure 14: Comparison with human coloring. Each bar shows how many of the human colorings belong to each tier in our scheme.

in the supplementary material. In order to measure the resemblance between human coloring and ours, we split the rank range $(1 \sim 120)$ into 12 sections (e.g. a rank from 1 to 10 belongs to the 1 st section), then identified where each human coloring ranked in our scheme. After analyzing 310 human colorings, we found that 107 of them belonged to the 1 st section and 66 to the second section, which means that more than half of the human colorings were within the top 20 ranks in our scheme (Figure 14). This high resemblance between human colorings and ours suggests the validity of basing our scheme on some established rules in visual perception and color theory. 

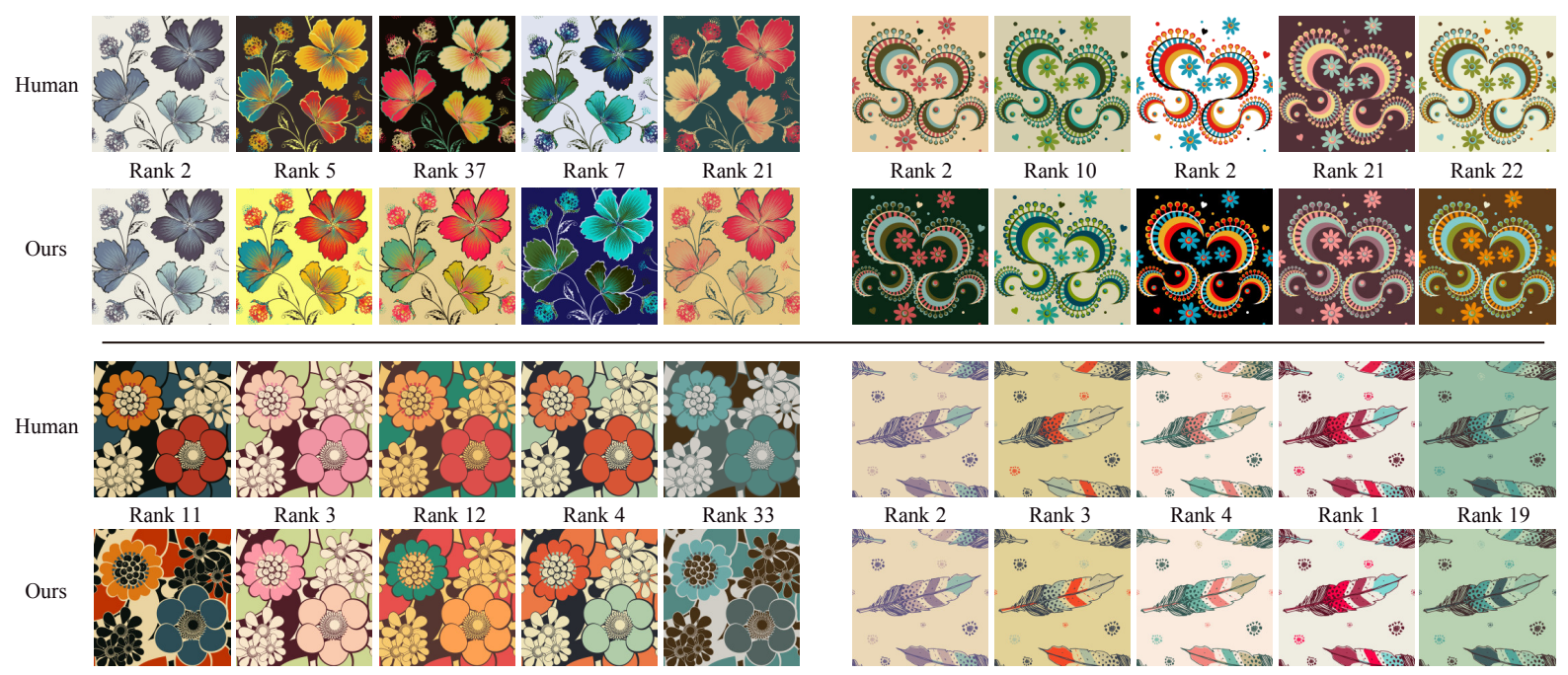

Figure 15: Comparison with human coloring. For each template, the top row (Human) shows top five human colorings and the bottom row (Ours) shows Rank 1 colorings by our method using the same color palettes suggested by the humans above. The numbers in the middle row denote where each human coloring ranks in our scheme.

\section{Discussion and Future work}

Our scheme allows multiple rules/constraints to compete and thus occasional conflicts may arise, although control parameters (such as $\lambda$ ) could help prioritize them somewhat. Another limitation is that our method does not take high-level semantics into account, and thus may assign unnatural colors to known objects (Figure 16), which could be problematic in certain applications.

Since we assume that the color palette is fixed, sometimes the deficiency in the input color palette itself may degrade the quality of the coloring result (Figure 17). A possible future research direction thus involves extending the current model to accommodate a flexible color palette that is capable of dynamically adjusting the colors in such a way that would improve the objective function value and therefore the quality of output.

Our framework may also be modified to incorporate additional features besides color and area, such as shape, orientation, and frequency. Another interesting future extension would be to develop an interactive colorization system that provides users with a visually intuitive control in some abstract parameter space.

\section{Appendix: Color harmony model}

$\mathrm{Ou}$ and Luo [OL06] proposed a color harmony function, $\mathcal{H}\left(Q_{1}, Q_{2}\right)$, for calculating harmony of two color combination $\left(Q_{1}, Q_{2}\right)$. The function is the sum of three terms:

$$
\mathcal{H}=H_{C}+H_{L}+H_{H} .
$$

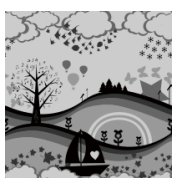

Template
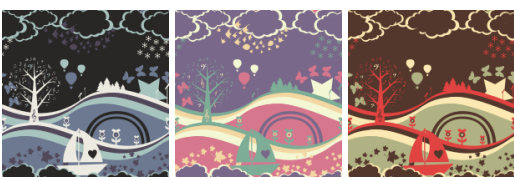

Rank 1
Figure 16: Our method does not consider image semantics and thus may assign unnatural colors to familiar scene elements, such as sky, sea, and trees.
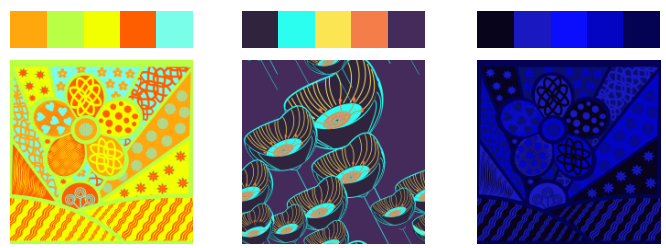

Figure 17: When the given palette provides insufficient harmony/contrast, or does not represent the content well.

The first term $H_{C}$ is given by

$$
\begin{aligned}
H_{C} & =0.04+0.53 \tanh (0.8-0.045 \Delta C), \\
\Delta C & =\left[\left(\Delta H_{a b}^{*}\right)^{2}+\left(\Delta C_{a b}^{*} / 1.46\right)^{2}\right]^{\frac{1}{2}},
\end{aligned}
$$

where $\Delta H_{a b}^{*}$ and $\Delta C_{a b}^{*}$ are the differences of hue and chroma values of $Q_{1}$ and $Q_{2}$ in CIELab color space. 
The second term $H_{L}$ is given by

$$
\begin{aligned}
H_{L} & =H_{\text {Lsum }}+H_{\Delta L}, \\
H_{\text {Lsum }} & =0.28+0.54 \tanh \left(-3.88+0.029 L_{\text {sum }}\right), \\
L_{\text {sum }} & =L_{1}^{*}+L_{2}^{*}, \\
H_{\Delta L} & =0.14+0.15 \tanh \left(-2+0.2 \Delta L_{12}\right),
\end{aligned}
$$

where $L_{1}^{*}$ and $L_{2}^{*}$ are lightness of $Q_{1}$ and $Q_{2} . \Delta L_{12}$ is the difference of them.

The third term $H_{H}$ is given by

$$
\begin{aligned}
H_{H}= & H_{S Y_{1}}+H_{S Y_{2}}, \\
H_{S Y_{i}}= & E_{C, i}\left(H_{S, i}+E_{Y, i}\right), \\
E_{C, i}= & 0.5+0.5 \tanh \left(-2+0.5 C_{a b, i}^{*}\right), \\
H_{S, i}= & -0.08-0.14 \sin \left(h_{a b, i}+50^{\circ}\right)-0.07 \sin \left(2 h_{a b, i}+90^{\circ}\right), \\
E_{Y, i}= & \left\{\left(0.22 L_{i}^{*}-12.8\right) / 10\right\} \exp \left[\left(90^{\circ}-h_{a b, i}\right) / 10\right. \\
& \left.-\exp \left\{\left(90^{\circ}-h_{a b, i}\right) / 10\right\}\right],
\end{aligned}
$$

where, $L_{i}^{*}$ represents the lightness of color $Q_{i}$, and $C_{a b, i}^{*}$ and $h_{a b, i}$ represent chroma, and hue angle of color $Q_{i}, i=1,2$, in CIELab color space, respectively.

\section{Acknowledgement}

This research is supported by Ministry of Culture, Sports and Tourism(MCST) and Korea Creative Content Agency(KOCCA) in the Culture Technology(CT) Research \& Development Program 2014 and the National Research Foundation of Korea(NRF) grant funded by the Korea government(MEST) (No. 2011-0028568).

\section{References}

[ADF10] Alexe B., Deselaers T., Ferrari V: What is an object? Computer Vision and Pattern Recognition, IEEE Conference on (2010), 73-80. 2

[Ado13] AdobeKuleR: https://kuler.adobe.com. Accessed in December 2013. 1, 5

[Bul07] Bullough E.: On the apparent heaviness of colours. British Journal of Psychology 2, 2 (1907), 111âĂŞ152. 4

[BVZ01] Boykov Y., Veksler O., Zabih R.: Fast approximate energy minimization via graph cuts. IEEE Transactions on Pattern Analysis and Machine Intelligence 23, 11 (2001), 1222 1239. 5

[COL13] COLOURLOVERS: https://www . colourlovers.com. Accessed in December 2013. 1, 2,5

[CoSG*06] Cohen-or D., Sorkine O., Gal R., Leyvand T., QING XU Y.: Color harmonization. ACM Transactions on Graphics 25, 3 (2006). 2

[CZM*11] Cheng M.-M., Zhang G.-X., Mitra N. J., HUANG X., HU S.-M.: Global contrast based salient region detection. Computer Vision and Pattern Recognition, IEEE Conference on (2011), 409-416. 2

[FGYZ12] Fu K., Gong C., YAng J., Zhou Y.: Salient object detection via color contrast and color distribution. Asian Conference on Computer Vision (2012), 374-381. 2
[GG84] Geman S., Geman D.: Stochatic relaxation, gibbs distributions, and the bayesian restoration of images. IEEE Transactions on Pattern Analysis and Machine Intelligence 6, 6 (1984), 721-741. 5

[Goe10] Goethe J.: Theory of Colours. 1810. 2

[Lam91] LAMMING D.: Contrast sensitivity. 4

[LRFH13] LIN S., RitchiE D., Fisher M., HANRAHAN P. Probabilistic color-by-numbers: Suggesting pattern colorization using factor graphs. ACM Transactions on Graphics 32, 4 (2013). $1,2,5$

[MI06] MAJUMder A., IRANi S.: Contrast enhancement of images using human contrast sensitivity. Proceeding APGV '06 Proceedings of the 3rd symposium on Applied perception in graphics and visualization (2006), 69-76. 2

[MLM13] Moretti G., Lyons P., Marsland S.: Computational production of colour harmony. part 1: A prototype colour harmonization tool. Color Research and Applications 38, 3 (2013), 203-217. 1, 2, 5

[MML13] Moretti G., Marsland S., Lyons P.: Computational production of colour harmony. part 2: Experimental evaluation of a tool for gui colour scheme creation. Color Research and Applications 38, 3 (2013), 218-228. 2

[MSK04] MeIER B. J., Spalter A. M., Karelitz D. B.: Interactive color palette tools. IEEE Computer Graphics and Applications 24, 3 (2004), 64-72. 1

[MZ03] MA Y.-F., ZHANG H.-J.: Contrast-based image attention analysis by using fuzzy growing. Proceeding MULTIMEDIA '03 Proceedings of the eleventh ACM international conference on Multimedia (2003), 374-381. 2, 4

[Nem07] NemCSICS A.: Experimental determination of laws of color harmony. part 1: Harmony content of different scales with similar hue. Color Research and Applications 32, 6 (2007), 477488. 2

[NS09] NaYATANi N., SAKai H.: Proposal for selecting twocolor combinations with various affections. part i: Introduction of the method. Color Research and Applications 34, 2 (2009), $128-134.2$

[OAH11] O'Donovan P., Agarwala A., Hertzmann A. Color Compatibility From Large Datasets. ACM Transactions on Graphics 30, 4 (2011). 1, 2, 3

[OL06] OU L.-C., Luo M. R.: A colour harmony model for two-colour combinations. Color Research and Applications 31, 3 (2006), 191-204. 2, 3, 9

[SBS10] Szabo F., Bodrogi P., Schanda J.: Experimental modeling of colour harmony. Color Research and Applications 35, 1 (2010), 34-49. 2, 3

[SMV*10] Sauvaget C., Manuel S., Vittaut J.-N., SUAREZ J., BOYER V.: Segmented images colorization using harmony. pp. 153-160. 1, 2

[SP11] Schloss K. B., PAlmer S. E.: Aesthetic response to color combinations: preference, harmony, and similarity. Attention, Perception ad Psychophysics (2011). 2

[Sto12] STONE M.: In color perception, size matters. Computer Graphics and Applications 32, 2 (2012), 8âĂŞ13. 5

[Wer38] WERTHEIMER M.: Laws of organization in perceptual forms. 1938. 5

[XZTZ10] Xu B., Zhuang Y., TANG H., Zhang L.: Objectbased multilevel contrast stretching method for image enhancement. IEEE Transactions on Consumer Electronics (2010), 1746-1754. 2 